\title{
When did round disk galaxies form?
}

\author{
T. M. Takeuchi, and K. Ohta \\ Department of Astronomy, Kyoto University, Kyoto 606-8502, Japan \\ ohta@kusastro.kyoto-u.ac.jp \\ S. Yuma \\ Institute for Cosmic Ray Research, The University of Tokyo, Kashiwa 277-8582, Japan \\ and \\ K. Yabe \\ National Astronomical Observatory of Japan, Mitaka 181- 8588, Japan
}

\begin{abstract}
When and how galaxy morphology such as disk and bulge seen in the present-day universe emerged is still not clear. In the universe at $z \gtrsim 2$, galaxies with various morphology are seen, and star-forming galaxies at $z \sim 2$ show an intrinsic shape of bar-like structure. Then, when did round disk structure form? Here we take a simple and straightforward approach to see the epoch when a round disk galaxy population emerged by constraining the intrinsic shape statistically based on apparent axial ratio distribution of galaxies. We derived the distributions of the apparent axial ratios in the rest-frame optical light $(\sim 5000 \AA)$ of star-forming main sequence galaxies at $2.5>z>1.4,1.4>z>0.85$, and $0.85>z>0.5$, and found that the apparent axial ratios of them show peaky distributions at $z \gtrsim 0.85$, while a rather flat distribution at the lower redshift. By using a tri-axial model $(A>B>C)$ for the intrinsic shape, we found the best-fit models give the peaks of the $B / A$ distribution of $0.81 \pm 0.04,0.84 \pm 0.04$, and $0.92 \pm 0.05$ at $2.5>z>1.4$, $1.4>z>0.85$, and $0.85>z>0.5$, respectively. The last value is close to the local value of 0.95 . Thickness $(C / A)$ is $\sim 0.25$ at all the redshifts and is close to the local value $(0.21)$. The results indicate the shape of the star-forming galaxies in the main sequence changes gradually, and the round disk is established at around $z \sim 0.9$. Establishment of the round disk may be due to a cease of violent interaction of galaxies or a growth of a bulge and/or a super-massive black hole resides at the center of a galaxy which dissolves the bar structure.
\end{abstract}

Subject headings: galaxies: evolution — galaxies: formation — galaxies: structure

\section{Introduction}

When and how did disk galaxies form? At $z \lesssim 1$, disk galaxies that can be classified with the Hubble's tuning fork exist (e.g., Schade et al. 1995; Abraham et al. 1996; Lilly et al. 1998; Scarlata et al. 2007; Sargent et al. 2007); they are identified with their apparent morphology, and/or their surface brightness distribution, and/or empirical indices describing such as central condensation and asym- metry. Meanwhile, in the universe at $z \gtrsim 3$, galaxies with various morphology are seen, and many of them show the presence of clumps and/or irregularity, though the morphology is traced in the rest-frame UV (e.g., Giavalisco et al. 1996; Steidel et al. 1996). Hence, the emergence of morphology classified with the Hubble's tuning fork is considered to be around at $z \sim 1-3$, and formation of disk structure would also be expected at the epoch. 
The Wide Field Camera 3 (WFC3) on the Hubble Space Telescope (HST) enables us to study morphology of galaxies in the rest-frame optical light well up to at $z \sim 3$. Conselice et al. (2011) studied morphology evolution based on eye inspection. They found the disk population is increasing with redshift gradually and the population is very rare at $z>2$. By using apparent morphology, similar trend is reported by Cameron et al. (2011) for galaxies with a stellar mass range of $10^{10-12} M_{\odot}$. and by Talia et al. (2014). Mortlock et al. (2013) also studied the morphology evolution with eye inspection and a clearer emergence of the disk population at $z \sim 2$ is seen after correcting for the statistical morphology misclassification.

However, from the optical surface brightness distribution, the fraction of the disk galaxies is higher at $z>2$ (Mortlock et al. 2013; Bruce et al. 2012; Buitrago et al. 2013). If the Sérsic index $(n)$ less than 2.5 is regarded to be an indicator of disk galaxies, the fraction of the disk population is larger at $z>2$ and is decreasing with redshift, though the details of the evolutional trend is not so simple; e.g., if irregular galaxies are included in the sample, the disk fraction is more constant against redshift (Buitrago et al. 2013), or the fraction is rather constant for less massive galaxies $\left(<10^{10.5} M_{\odot}\right)$ (Mortlock et al. 2013). Bruce et al. (2012) tried to model the two-dimensional surface brightness distribution with two components of Sérsic index of $n=1$ and $n=4$, and found a fraction of the disk ( $n=1$ component) dominated galaxies decreases at $z \sim 2$ rather sharply for stellar mass larger than $10^{10.5} M_{\odot}$.

Thus the opposite views are obtained in terms of the disk evolution. Other parameters characterizing the morphological features such as central condensation, asymmetry, Gini, M20, etc seem not to be suitable to trace the morphology of galaxies in distant universe (Wang et al. 2012; Mortlock et al. 2013; Talia et al. 2014).

On the other hand, studies of internal kinematics in star-forming galaxies such as BM/BX and star-forming $\mathrm{BzK}(\mathrm{sBzK})$ galaxies at $z \sim 2$ revealed about one third of them show a clear rotation kinematics (Förster Schreiber et al. 2009). This suggests we witness disk galaxies and they may be progenitor of present-day disk galaxies, though a fraction of them may evolve into the present-day elliptical galaxies through ma- jor merges, particularly those with large stellar mass. Another piece of the suggestion for the disk progenitor scenario stems from the clustering property of sBzK galaxies; sBzK galaxies with $K<23$ mag are considered to be a progenitor of present-day disk galaxies with regard to their weak clustering strength (Hayashi et al. 2007). It would be reasonable to suppose most of the sBzK galaxies in this magnitude range will evolve into the present-day disk galaxies, though the brighter sBzK galaxies may be a progenitor of elliptical galaxies.

Motivated by the finding of the presence of many rotating disk among sBzK galaxies and clustering nature of them, we took a simple and straightforward approach to see whether the intrinsic shape of faint sBzK galaxies at $z \sim 2$ is disk-like or not statistically based on apparent axial ratio distribution. We studied the surface brightness distribution and the apparent axial ratio in both rest-frame UV and optical light for sBzK galaxies with $K_{\mathrm{S}}<24 \mathrm{mag}$ (Yuma et al. 2011, 2012). Yuma et al. (2011) and Yuma et al. (2012) revealed the surface brightness distribution of them is characterized by Sérsic index of $n \sim 1$, and the half-light radius and surface stellar mass density is similar to those in the present-day disk galaxies (Barden et al. 2005). However, it was turned out that the intrinsic shape of the sBzK galaxies is not a round disk (round means intrinsic axial ratio of $\sim 0.9-1.0)$; their intrinsic shape is bar-like or oval (intrinsic axial ratio $\sim 0.6-0.8$ ) with the thickness similar to that of the presentday disk galaxies. The intrinsic bar-like structure of star-forming galaxies at $z \sim 2$ is also pointed out by Law et al. (2012). It should be noted here that this bar-like structure does not imply a direct progenitor of the local barred galaxies as discuss later.

Then, when did round disk galaxies appear after $z \sim 2$ ? Emergence of the round disk population should reflect the evolution process of the disk structure. Hence, revealing evolution of the intrinsic shape of the galaxies is expected to give us an insight to physical process of the galaxy evolution. In this paper, we study the intrinsic shape of main sequence galaxies with $K_{\mathrm{S}}<24$ mag in the redshift range from 2.5 to 0.5 using the same method as Yuma et al. (2011) and Yuma et al. (2012) to see the redshift that the round disk galaxy pop- 
ulation emerged. In the subsequent section, data sources and sample galaxies are summarized. In $\S 3$, we derive the surface brightness distributions and axial ratios of the galaxies. Then, in $\S 4$, we examine the distributions of the apparent axial ratios and constrain the intrinsic shape of the galaxies. Conclusion and discussion are given in $\S 5$. We adopt a cosmology with $H_{0}=70 \mathrm{~km} \mathrm{~s}^{-1} \mathrm{Mpc}^{-1}$, $\Omega_{\mathrm{m}}=0.3$, and $\Omega_{\Lambda}=0.7$. All magnitudes in this paper are given in the $\mathrm{AB}$ magnitude system.

\section{Data sources and sample galaxies}

Sample galaxies in this study are collected from the Great Observatories Origins Deep SurveySouth (GOODS-S) 1 (Dickinson et al. 2003) and the Subaru XMM-Newton Deep Survey (SXDS) (Furusawa et al. 2008), which almost overlaps Ultra Deep Survey (UDS) in UK Infrared Telescope Infrared Deep Sky Survey (UKIDSS) Lawrence et al. 2007). The data sources are the same as those used by Yuma et al. (2012) and the details are described in the paper. Here we briefly summarize them. In GOODS-S region, near infrared (NIR) images $\left(J_{-}, H-\right.$, and $K_{\mathrm{S}}$-band images) were taken from GOODS/ISAAC (Infrared Spectrometer And Array Camera) data release final version (Retzlaff et al. 2010). Five sigma limiting $K_{\mathrm{S}}$-band magnitude is $24.4 \mathrm{mag}$. NIR images taken with WFC3 on $H S T$ with F125W and F160W filters were obtained from CANDELS (Cosmic Assembly Near-infrared Deep Extragalactic Legacy Survey; Grogin et al. 2011; Koekemoer et al. 2011)2 data release v0.5. Optical images were taken from ver. 2.0 data products of GOODS HST /ACS(Advanced Camera for Surveys) treasure program $\left(B_{435}, V_{660}, i_{775}\right.$, and $\left.z_{850}\right)$ (Giavalisco et al. 2004) and from ESO/GOODS program $(U$ and $R$ ) (Nonino et al. 2009). Midinfrared (MIR) images at $3.6 \mu \mathrm{m}$ and $4.5 \mu \mathrm{m}$ taken with Infrared Array Camera (IRAC) on the Spitzer Space Telescope (SST) were from data release 1 and 2 obtained in Spitzer Legacy Science program. In SXDS/UDS region, NIR images $\left(J, H\right.$, and $\left.K_{\mathrm{S}}\right)$ were taken from data release ver. 8 of UKIDSS UDS. Five sigma limiting magnitude is $K_{\mathrm{S}}=24.6 \mathrm{mag}$. NIR images with HST/WFC3 with F125W and F160W filters were

1 http://www.sci.edu/science/goods

2 http://candels.ucolick.org/index.html obtained from CANDELS data release v1.0. Optical images were taken from SXDS project $(B$, $V, R_{\mathrm{C}}, i^{\prime}$, and $z^{\prime}$ ) (Furusawa et al. 2008). We used $H S T / \mathrm{ACS}$ images taken with F814W filter $\left(i_{814}\right)$. MIR $3.6 \mu \mathrm{m}$ and $4.5 \mu \mathrm{m}$ images were taken from Spitzer Public Legacy Survey of UKIDSS UDS (SpUDS; PI: J. Dunlop). We also use the far-UV and near-UV data taken from Galaxy Evolution Explorer (GALEX; Martin et al. 2005) archived image (GR6) and the $u$-band data of the Canada-France-Hawaii Telescope Legacy Survey (CFHTLS) wide field as supplemental information, though the limiting magnitudes are shallow.

Object detection was made based on $K_{\mathrm{S}}$-band image by using SExtractor Bertin \& Arnouts 1996). We made photometry using these data for each source detected in $K_{\mathrm{S}}$ images at the same position and derived total magnitudes using SExtraCtor. For the images taken with ACS and IRAC, we applied a correction for the aperture photometry to obtain a total magnitude following the manner by Yuma et al. (2012). Spectral energy distribution (SED) was constructed for each object from the photometry in all the bands. Then photometric redshifts were derived by using HYPERz (Bolzonella et al. 2000). Comparisons with spectroscopic redshifts available showed $\Delta z /(1+z)$ of $\sim 0.05$ and $\sim 0.03$ in GOODS-S and SXDS, respectively. With the photometric redshifts, stellar mass, star-formation rate (SFR), and color excess $(E(B-V))$ were derived for each object through SED fitting by using SEDfit program (Sawicki 2012). The SEDfit employs population synthesis code of BC03(Bruzual \& Charlot 2003). Salpeter initial mass function with a mass range of $0.1-100 M_{\odot}$ and the solar metallicity were assumed. Extinction law used was that by Calzetti et al. (2000). More details are described by Yuma et al. (2011, 2012) and Yabe et al. (2012, 2014).

Using these data, we selected galaxies brighter than $K_{\mathrm{S}}=24.0 \mathrm{mag}$. We divided the galaxies in three epochs of $2.5>z>1.4,1.4>z>0.85$, and $0.85>z>0.5$ based on the photometric redshifts; these epochs are chosen to take a comparable duration ( $\sim 2$ Gyr) of the epochs in the cosmic age and to keep a reasonable sample size.

Figure 1 shows distribution of the SFR against stellar mass in each epoch. As like in other works (e.g., Noeske et al. 2007; Daddi et al. 2007), the SFRs are larger in more massive galaxies and they 
make a sequence referred as main sequence. The main sequence shows a gradual cosmological evolution down to $z \sim 0$, where we see disk galaxies in the sequence. Most of our sample galaxies at $2.5>z>1.4$ are also sBzK galaxies. Galaxies in the most massive part of the main sequence at the higher redshifts may soon stop star formation and evolve into passive elliptical galaxies according to a simple theoretical expectation (e.g., Bouché et al. 2010). But most of the sample galaxies occupy less massive part of the main sequence and they are expected to reside in the main sequence at the present epoch. A branch seen above the main sequence shows very high SFR at a fixed stellar mass, and they are considered to be violently forming stars often under strong galaxy interaction (e.g., Daddi et al. 2007). Thus we selected star-forming galaxies in the main sequence bracketed by thresholds shown in Figure 1 The stellar mass limit at $5 \times 10^{9} M_{\odot}$ is to avoid the inclusion of Magellanic-type galaxies that would tend to show bar-like structure even in the local universe. A star-forming galaxy with a stellar mass of $\sim 5 \times 10^{9} M_{\odot}$ at $z=2.5$ is a progenitor of the Milky Way class galaxy (stellar mass of $5 \times 10^{10} M_{\odot}$ ), if we adopt the abundance matching in terms of the number density (van Dokkum et al. 2013). Ranges of the specific SFR (sSFR) we use are $3 \times 10^{-11}-2 \times 10^{-8}$ $\mathrm{yr}^{-1}(z=1.4-2.5), 3 \times 10^{-11}-1 \times 10^{-8} \mathrm{yr}^{-1}$ $(z=0.85-1.4)$, and $2 \times 10^{-11}-3 \times 10^{-9} \mathrm{yr}^{-1}$ $(z=0.5-0.85)$. SFR limit is just an additional threshold; SFR $>2.0 M_{\odot} \mathrm{yr}^{-1}(z=1.4-2.5)$, $>0.6 M_{\odot} \mathrm{yr}^{-1}(z=0.85-1.4)$, and $>0.2 M_{\odot} \mathrm{yr}^{-1}$ $(z=0.5-0.85)$. A fraction (typically $10-15 \%)$ of them show multiple-structure in the rest-frame optical HST images; if an object detected by SEXTRACTOR locates within a radius of median size (FWHM) of the galaxies in each epoch, we regard them as multiple. Such galaxies are not adequate to examine the shape and we discarded them in this study.

\section{Two-dimensional surface brightness fit- ting}

We made two-dimensional surface brightness distribution fitting to the galaxies selected above. The images were taken with $H S T$ in the restframe optical wavelength $(\sim 0.5 \mu \mathrm{m}): \mathrm{F} 160 \mathrm{~W}\left(H_{160}\right)$ for $2.5>z>1.4, \mathrm{~F} 125 \mathrm{~W}\left(J_{125}\right)$ for $1.4>z>0.85$, and $\mathrm{F} 850 \mathrm{LP}\left(z_{850}\right)$ in GOODS-S and $\mathrm{F} 814 \mathrm{~W}\left(i_{814}\right)$ in SXDS for $0.85>z>0.5$. Magnitude ranges for the sample galaxies in three epochs were virtually $H_{160}<24 \mathrm{mag}$ and $J_{125}<24 \mathrm{mag}$, and $z_{850}<25$ mag in GOODS-S and $i_{814}<24$ mag in SXDS. Pixel scales after drizzle were $0 .{ }^{\prime \prime} 06,0 .{ }^{\prime \prime} 06$, and $0 .{ }^{\prime \prime} 03$ in $H_{160}, J_{125}$, and $z_{850} / i_{814}$, respectively.

The radial distribution is fitted with Sérsic profile: $I(r)=I_{e} \exp \left[-\kappa_{n}\left\{\left(r / r_{e}\right)^{1 / n}-1\right\}\right]$, where $r_{e}$ is the half light radius, $I_{e}$ is the surface brightness at $r_{e}$, and $n$ characterizes the shape of the profile (Sérsic index). Two dimensional surface brightness fitting was made by using GALFIT version 3 (Peng et al. 2010). Initial guess parameters on the central position, axial ratio, position angle, and $r_{e}$ are taken from the output parameters of SExtractor. Initial Sérsic index was set to be 1.5 , which does not affect the final results significantly. Point spread function in each image was constructed from $5 \sim 20$ isolated unsaturated stars.

Figure 2 shows example of the profile fitting. As seen in the figure, the surface brightness profiles extend to $\sim 1$ arcsec in radius corresponding to $\sim 8 \mathrm{kpc}$ at $z=1 \sim 2$ and $\sim 6 \mathrm{kpc}$ at $z=0.5$. The surface brightness we use to fit the model reaches $23.5 \sim 25 \mathrm{mag} \operatorname{arcsec}^{-2}$ in the restframe $V$ band after correcting for the cosmological dimming. This level of the surface brightness can be regarded as the outskirt of galaxies, and is deep enough to confront with the shape of local disk galaxies. Considering the fading of galaxy luminosity to the present epoch, the value would be $\sim 1 \mathrm{mag} \operatorname{arcsec}^{-2}$ fainter at $z \sim 0$ (e.g., Brinchmann et al. 1998; Miller et al. 2011). We obtained $r_{e}, n$, and apparent axial ratio $(b / a$, where $a$ is a major axis and $b$ is a minor axis) for each galaxy. About $60-70 \%$ of the sample galaxies show $n=0.5-2.5$. We then examined the distribution of $r_{e}$ and stellar mass of the sample galaxies with $n=0.5-2.5$. In each epoch, the distribution of $r_{e}$ against stellar mass is similar to that for local disk galaxies (Barden et al. 2005).

Accuracy for the Sérsic index as well as for the axial ratio was examined by putting artificial objects into the images used for the analysis. The artificial objects were created by using ARTDATA/MKOBJECTS in IRAF. Mock galaxies with Sérsic index of 1 or 4 were generated in both GOODS-S and SXDS with half-light radius 

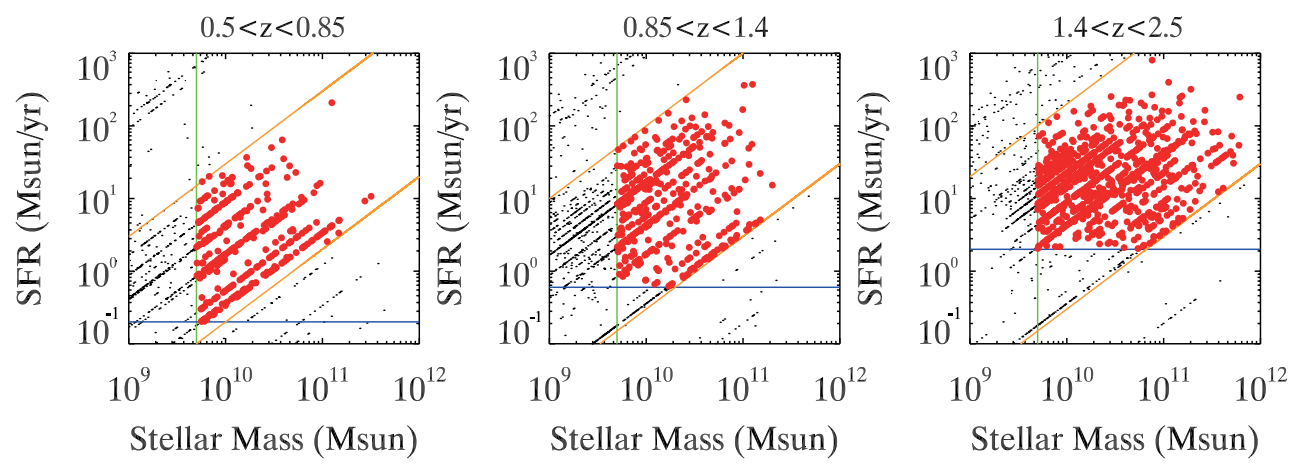

Fig. 1. - Star formation rate versus stellar mass of the sample galaxies at three epochs: From right to left, $2.5>z>1.4,1.4>z>0.85$, and $0.85>z>0.5$. Large red points are sample in this study and solid lines in each panel show the selection criteria

(see text).

of $0 . .^{\prime \prime} 1-1 .^{\prime \prime} 0$ in a magnitude range of $20-25$ mag. The ranges of input parameters are chosen to mimic those of the sample galaxies. Axial ratio and position angle are randomly chosen and the galaxies are put into $H_{160}, J_{125}, z_{850}$, and $i_{814}$ images randomly. As we did for the real objects, we first used SExtractor to derive position, position angle, axial ratio, and FWHM. We then performed GALFIT with these parameters as well as $n=1.5$ as the initial guess. Results for $H_{160}$ (GOODS-S, SXDS/UDS) were presented by Yuma et al. (2012). Accuracy for the Sérsic index in $H_{160}$ image is good enough $(<10 \%)$ in this magnitude range in case of $n=1$. Even if the intrinsic $n$ is 4 , the misclassification into $n=1$ disk is negligible, though the accuracy is slightly worse in $z_{850}$ image (Yuma et al. 2011, 2012). We show the accuracy of the axial ratio derived for $J_{125}$ (SXDS/UDS), $z_{850}$ (GOODS-S), and $i_{814}$ (SXDS/UDS) in case of $n=1$ in Figure 3, In all the panels, the obtained axial ratios agree very well with those of input value in the bright magnitude range, and the accuracy is getting worse for the fainter objects. However, at the faintest magnitude level of the sample galaxies in each band, the accuracy of the obtained axial ratio is less than $5 \%$ on average, which is smaller than the bin size of the histograms of the axial ratio distribution described below. The accuracy of axial ratio in $H_{160}$ (GOODS-S, SXDS/UDS) is presented by Yuma et al. (2012), and it is comparable to those in other bands. Detection rate in $K_{\mathrm{S}}$-band image against the apparent axial ratio was also exam- ined and the rate is almost constant in the range of $b / a=0.2$ and 1.0 (Yuma et al. 2011).

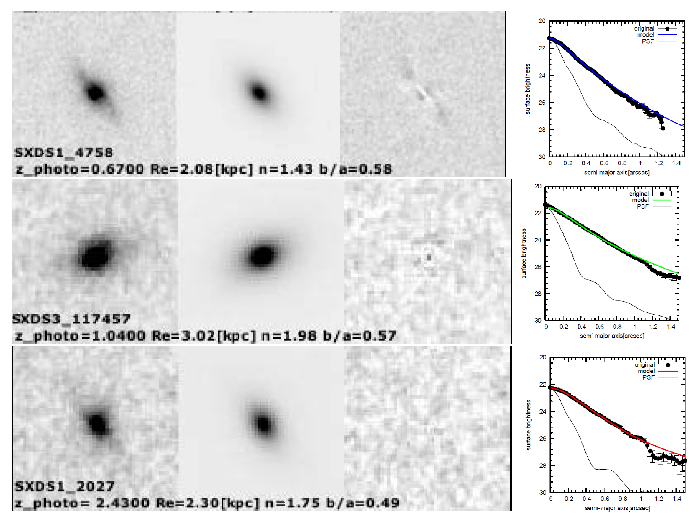

Fig. 2.- Example of the surface brightness distribution fitting at $0.85>z>0.5$ (upper), $1.4>$ $z>0.85$ (middle), and $2.5>z>1.4$ (lower). The images are taken with $H S T i_{814}$ (upper), WFC3 $J_{125}$ (middle), and WFC3 $H_{160}$ (lower). In each row, from left to right, original image, best-fit model image, residual, and radial surface brightness profiles along the major axis are shown. In the rightmost panel, the observed surface brightness and the best-fitted profile smoothed with point spread function is shown with circles with error and solid curve, respectively. Point spread function is shown with thin solid curve. 


\section{Axial ratio distribution}

\subsection{Distribution of apparent axial ratios}

In order to see whether they have round disk structure, we take a simple and straightforward approach; i.e., we constrain the intrinsic shape of these galaxies statistically based on distribution of apparent axial ratios of the sample galaxies at three epochs. Figure 4 (upper row) shows the apparent axial ratio distributions of the sample galaxies with $n=0.5-2.5$ among galaxies selected in 92. If the intrinsic shape of disk galaxies is an infinitesimally thin circular disk, the apparent axial ratio distribution is exactly flat. In fact, the distribution for $\sim 300,000$ local disk galaxies is almost flat except for around at $b / a \sim 0$ and $\sim 1$ (Padilla and Strauss 2008), since real disks have a finite thickness and are not perfectly round. As seen in the right panel $(2.5>z>1.4)$ of the upper row of Figure 4, the axial ratio distribution peaks at $\sim 0.45$ and sharply decreases to both sides. In the middle panel $(1.4>z>0.85)$, the distribution is slightly flatter, but still shows a slight peaky distribution. The histograms suggest these galaxies do not have a round disk structure. Meanwhile in the left panel $(0.85>z>0.5)$, the distribution is rather flat and is close to that for the local disk galaxies, suggesting they have the intrinsic shape of round disk.
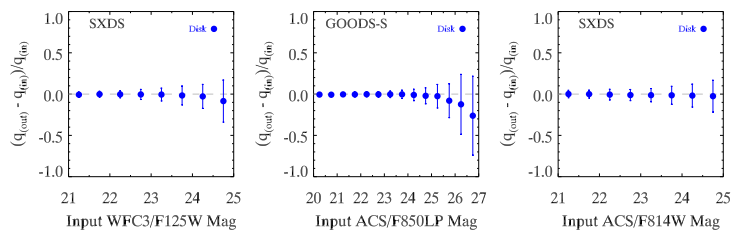

Fig. 3.- Accuracy of the obtained axial ratio against magnitude of input artificial object in $J_{125}$ (left), $z_{850}$ (middle), and $i_{814}$ (right). Error bars represent $1 \sigma$ dispersion of the distribution.

\subsection{Intrinsic axial ratio distribution}

To constrain the intrinsic shape quantitatively, we employ a tri-axial model with axial lengths of $A>B>C$ following the method by Ryden (2004). The face-on ellipticity $(\epsilon=1-B / A)$ is assumed to be described by log-normal distribution with mean of $\mu(=\ln \epsilon)$ and dispersion of $\sigma$, while the edge-on thickness $(C / A)$ is assumed to be described with Gaussian distribution with mean of $\mu_{\gamma}$ and dispersion of $\sigma_{\gamma}$. With this parameter set, the expected apparent axial ratio distribution seen from random viewing angles can be calculated and can be compared with the observed apparent axial ratio distribution.

The model axial ratio distributions were calculated in a range of $\mu=-3.95 \sim-0.05, \sigma=$ $0.2 \sim 2.0, \mu_{\gamma}=0.1 \sim 0.98$, and $\sigma_{\gamma}=0.01 \sim 0.35$, with a step of $\Delta \mu=0.15, \Delta \sigma=0.15, \Delta \mu_{\gamma}=0.02$, and $\Delta \sigma_{\gamma}=0.02$. With these parameter sets, we searched for the best-fit parameter set using $\chi^{2}$ method with $1 \sigma$ statistics by Gehrels (1986). To estimate the uncertainty of the best-fit parameters, we made Monte Carlo realization of the observed histogram of the axial ratio 1000 times and derived the best-fit parameter set for each realization. We took $68 \%$ confidence level as the uncertainty of the model parameter. The peak value of the distribution of intrinsic $B / A$ is given by $1-\exp \left(\mu-\sigma^{2}\right)$. The uncertainty on the peak $B / A$ was calculated based on the propagation of errors with the uncertainty of $\mu$ and $\sigma$ mentioned above.

The best-fit models are shown with solid curves in Figure 4 (upper row), and the best-fitted parameters as well as the uncertainties are shown in Table 1. Resulting distributions of intrinsic axial ratios at the epochs are shown in Figure 4 (bottom row). The best-fit model $B / A$ peaks at $0.81 \pm 0.04,0.84 \pm 0.04$, and $0.92 \pm 0.05$ at $2.5>z>1.4,1.4>z>0.85$, and $0.85>z>0.5$, respectively, and the last value of $B / A$ is very close to that for the local disk galaxies (0.94) (Padilla and Strauss 2008). The mean $C / A$ is $\sim 0.25$ at all the epochs and is close to that of the local disk galaxies (0.21) (Padilla and Strauss 2008). The intrinsic shape of the star-forming main sequence galaxies at $2.5>z>0.85$ is barlike or oval, while they have round disk structure at $z<0.85$, i.e., the emergence of the round disk population is $z \sim 0.9$, though the precise redshift determination is difficult due to the the gradual cosmological evolution and small sample size.

\subsection{Robustness of the results}

In order to examine the robustness of the results, we constructed samples by changing selection criteria slightly with respect to $K_{\mathrm{S}}$-band magnitude, stellar mass, sSFR, $i_{814} / z_{850} / J_{125} / H_{160}$ 


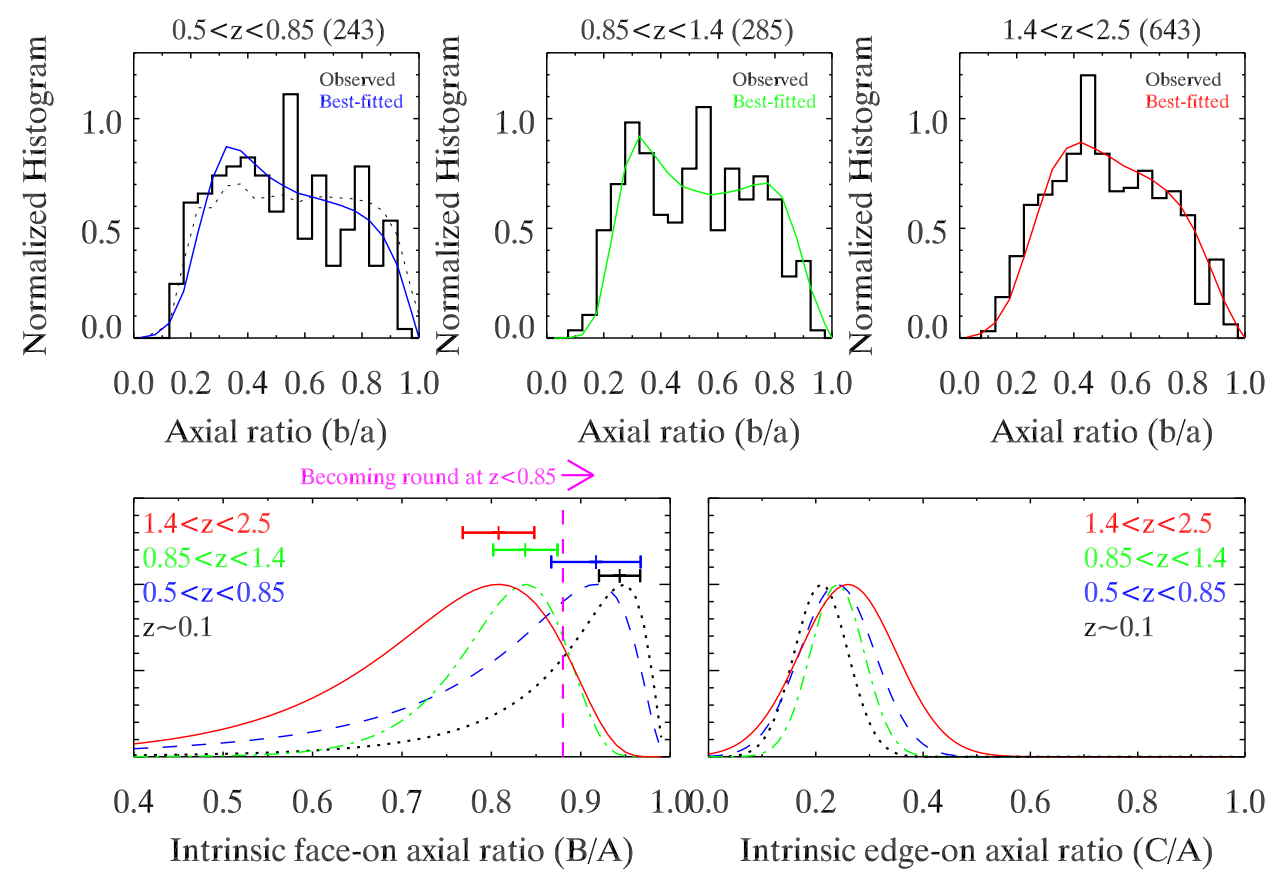

Fig. 4.- Normalized histograms of the observed axial ratios at each epoch (upper panels):From right to left, $2.5>z>1.4,1.4>z>0.85$, and $0.85>z>0.5$. Numbers in vertical axis are multiplied by 10 . Number in the parenthesis at the top of each panel refers to the sample size. Best-fit model distributions are shown with solid curves. Axial distribution of local disk galaxies from the Sloan Digital Sky Survey Data Release 6 by Padilla \& Strauss (2008) is also shown with dotted curve (left panel). Distribution of best-fit intrinsic axial ratios of $B / A$ and $C / A$ (bottom panels). Red solid, green dot dashed, and blue dashed curves refer to the intrinsic model axial distribution at $2.5>z>1.4,1.4>z>0.85$, and $0.85>z>0.5$, respectively. Black dotted curves show the distribution for local disk galaxies (Padilla and Strauss 2008). Y-axis is an arbitrary unit. Peaks for the $B / A$ distribution are shown by tick marks with uncertainty above the curves.

Table 1: Best-fit parameter sets for the tri-axial model.

\begin{tabular}{ccccc}
\hline \hline & Local $^{*}$ & $z=0.5-0.85$ & $z=0.85-1.4$ & $z=1.4-2.5$ \\
\hline$\mu$ & $-2.33_{-0.13}^{+0.13}$ & $-1.85_{-0.18}^{+0.35}$ & $-1.70_{-2.30}^{+0.12}$ & $-1.40_{-0.08}^{+0.08}$ \\
$\sigma$ & $0.79_{-0.16}^{+0.16}$ & $0.80_{-0.60}^{+0.19}$ & $0.35_{-0.13}^{+0.19}$ & $0.50_{-0.18}^{+0.17}$ \\
$\mu_{\gamma}$ & $0.21_{-0.02}^{+0.02}$ & $0.24_{-0.02}^{+0.02}$ & $0.24_{-0.01}^{+0.01}$ & $0.26_{-0.16}^{+0.02}$ \\
$\sigma_{\gamma}$ & $0.050_{-0.015}^{+0.015}$ & $0.07_{-0.06}^{+0.16}$ & $0.05_{-0.01}^{+0.01}$ & $0.09_{-0.08}^{+0.01}$ \\
\hline
\end{tabular}

*Padilla \& Strauss (2008)

magnitude, and Sérsic index, and made the same fitting to these samples.

If we take samples in each epoch with $0.5 \mathrm{mag}$ brighter or fainter in $K_{\mathrm{S}}$ band, virtually all the best-fit parameters are within $1 \sigma$ error. The peak values of the intrinsic axial distribution $(B / A)$ agree with the best-fit value within $1 \sigma$ error. If we take a massive sample (stellar mass larger than $\left.1.0 \times 10^{10} M_{\odot}\right)$, the results mostly agree within 1 $\sigma$ error. If we include less massive galaxies (stellar mass larger than $3 \times 10^{9} M_{\odot}$ ), peak values of $B / A$ agree within the error, but the resulting parame- 
ters for the highest-redshift bin change more than $1 \sigma$. It should be noted that even in local universe such less massive galaxies often show barlike structure as like Magellanic irregular galaxies. We also test the dependence on sSFR by taking $3 \times 10^{-11} \mathrm{yr}^{-1}<\mathrm{sSFR}<2 \times 10^{-8} \mathrm{yr}^{-1}$ at $z=2.5-1.4,3 \times 10^{-11} \mathrm{yr}^{-1}<\mathrm{sSFR}<5 \times 10^{-9}$ $\mathrm{yr}^{-1}$ at $z=1.4-0.85$, and $3 \times 10^{-11} \mathrm{yr}^{-1}<\mathrm{sSFR}$ $<7 \times 10^{-9} \mathrm{yr}^{-1}$ at $z=0.85-0.5$. Almost all the obtained best-fit parameters as well as the peak values of $B / A$ agree within $1 \sigma$ error. If we take the threshold SFR 2-3 times larger, the resulting parameters are almost within $1 \sigma$. The axial ratios are derived by using $i_{814}, z_{850}, J_{125}$, and $H_{160}$ images. More accurate axial ratios are expected to be obtained with the brighter sample. Hence we take about 0.5 mag brighter samples $\left(i_{814}<\right.$ $23.5 \mathrm{mag}, z_{850}<24.5 \mathrm{mag}, J_{125}<23.5 \mathrm{mag}$, and $\left.H_{160}<23.5 \mathrm{mag}\right)$ from the $K_{\mathrm{S}}<24.0 \mathrm{mag}$ sample. The resulting peak axial ratios are within or marginally within $1 \sigma$ uncertainty, and the conclusion in this study does not change. We also make samples with Sérsic index of $0.5-1.5$ and $0.5-3.5$, and find again the conclusion does not change.

Dust obscuration may affect the axial ratio distribution; the redder disk galaxies tend to show the smaller axial ratios in the local universe as well as at $z=0.6-0.9$ (e.g., Padilla and Strauss 2008; Patel et al. 2012). This trend is considered to be due to the larger dust obscuration in more inclined galaxies. In order to see the effect, we divided the sample into two subsamples with smaller or larger color excess $(E(B-V))$ at each epoch. The color excess was derived from the SED fitting mentioned in $\S 2$, and we divided the sample at around the median $E(B-V)$ of 0.2 or 0.25 mag. Resulting histograms of the axial ratios show that a slight excess at smaller axial ratio $(b / a \sim 0.2-0.4)$ is seen at $z=0.5 \sim 0.85$, but such a clear trend is not seen at the higher redshifts. We made the model fitting to the subsamples. About half of the best fit $\mu$ and $\sigma$ values agree with those of the total sample within the uncertainty for the total sample. However, it should be noted that the uncertainty for the subsamples is large due to the smaller sample size. We also derived the peak $B / A$ with the subsamples. The peak $B / A$ values in the smaller color excess (larger color excess) are $0.81(0.93)$ at $2.5>z>1.4,0.91(0.78)$ at $1.4>z>0.85$, and $0.99(0.97)$ at $0.85>z>0.5$. Thus the increas- ing trend of $B / A$ with decreasing $z$ is still seen, except for the $B / A$ for the larger color excess at $2.5>z>1.4$.

\section{Conclusion and discussion}

We constrain the intrinsic shape of the starforming main sequence galaxies at $z=2.5$ to 0.5 based on the distributions of the apparent axial ratios by employing the tri-axial model of $A>B>C$. At $z \gtrsim 0.9$, the intrinsic shape of them is bar-like or oval with $B / A \sim 0.8$, while at $z \lesssim 0.9, B / A$ is 0.92 that is close to the value for the local galaxies (0.95). Hence, the emergence of the round disk can be regarded to be $z \sim 0.9$, though the precise redshift determination is difficult due to the gradual evolution and the small sample size. The thickness $(C / A)$ is about 0.25 and does not show significant cosmological evolution down to $z \sim 0$. Similar result has been obtained very recently by adopting a slightly different model for the intrinsic shape; the triaxiality of star-forming galaxies with stellar mass larger than $10^{9.5} M_{\odot}$ is large at $z \gtrsim 1$, but small at $z \lesssim 1$ van der Wel et al. 2014). It is worth noting the bar-like structure we see at the high redshifts is unlikely to be a direct progenitor of bar structure in the present-day barred galaxies, because the apparent axial ratios are obtained mainly from outside beyond the bar region in the present-day barred galaxies; the bar region in a present-day barred galaxy is brighter than $\sim 22 \mathrm{mag} \operatorname{arcsec}^{-2}$ in $V$ band (Ohta et al. 1990, 2007). Furthermore, large barred galaxies are disappearing as redshift increases (van den Bergh et al. 1996): Its fraction among disk galaxies is only $10-20 \%$ at $z=0.8 \sim 1.0$ (Sheth et al. 2008; Melvin et al. 2014).

At $z \gtrsim 3$, most of star-forming galaxies show Sérsic index of $\sim 1$ in the rest-frame UV (Ravindranath et al. 2006) and in the rest-frame optical (Akiyama et al. 2008). However, the distribution of the apparent axial ratios is not flat, suggesting the elongated intrinsic structure (Ravindranath et al. 2006). The results obtained here reveal that although the disk-like structure is seen among star-forming galaxies at $z \gtrsim 1$, its intrinsic shape is bar-like or oval. Physical mechanism to make such structure is not clear, but they may be bar-like due to galaxy 
interaction and/or minor merge (Noguchi 1987; Berentzen et al. 2004). Or the baryonic matter might have been already highly concentrated in a dark matter halo, resulting into the bar instability (Ostriker \& Peebles 1973).

Then how did they become round? Numerical simulations show a central mass condensation (either of a bulge and/or a super-massive black hole $(\mathrm{SMBH}))$ with a mass fraction of 1$10 \%$ of a disk dissolves the bar-like structure (Athanassoula et al. 2005; Hozumi 2012). Thus the emergence of round disk population may be related to the growth of a central bulge and/or a SMBH. According to the simulations by Athanassoula et al. (2005), the bar-like structure gradually evolves into a round disk with a time scale of a few Gyr, which is comparable to the elapsed time from $z=1.5$ to 0.9 ( $\sim 2 \mathrm{Gyr})$. However, eye inspection of our sample galaxies at $z=0.85-0.5$ suggests that bulge dominated galaxies are not the majority. The two-component model fit to discriminate bulge and disk in a galaxy is not straightforward and it is beyond our scope in this study. AGN number density peaks at $z=1-2$ (e.g., Ueda et al. 2003; Hasinger et al. 2005), suggesting the rapid growth of SMBHs during the epoch. The growth of a SMBH may also play a role for dissolving the bar structure, although the mass fraction may not be large enough. Alternatively, the gradual cease of galaxy interaction may make a round disk. If this is the case, the epoch of the round disk population at $z \sim 0.9$ would indicate the major galaxy interaction mostly ceased around at this redshift and the galaxy morphology is rather stable after this. Further studies of link between intrinsic shape of disk and the growth of a bulge/SMBH as well as galaxy interaction are desirable to disclose the evolution of disk galaxies.

We thank the referee for the useful comments. KO's efforts are supported by the Grant-in-Aid for Scientific Research (24540230) and the Grantin-Aid for Scientific Research on Innovative Area (24103003) from the Japan Society for the Promotion of Science. This work is partially based on observations taken by the CANDELS Multi-Cycle Treasury Program with the NASA/ESA HST, which is operated by the Association of Universities for Research in Astronomy, Inc., under NASA contract NAS5-26555. Parts of the observations in GOODS-S have been carried out using the Very Large Telescope at the ESO Paranal Observatory under Program ID 168.A-0485. Based on observations obtained with MegaPrime/MegaCam, a joint project of CFHT and CEA/IRFU, at the CanadaFrance-Hawaii Telescope (CFHT) which is operated by the National Research Council (NRC) of Canada, the Institut National des Science de l'Univers of the Centre National de la Recherche Scientifique (CNRS) of France, and the University of Hawaii. This work is based in part on data products produced at Terapix available at the Canadian Astronomy Data Centre as part of the Canada-France-Hawaii Telescope Legacy Survey, a collaborative project of NRC and CNRS.

\section{REFERENCES}

Abraham, R. G., Tanvir, N. R., Santiago, B. X., et al.(1996), MNRAS, 279, L47

Akiyama, M., Minowa, Y., Kobayashi, N., et al. 2008, ApJS, 175, 1

Athanassoula, E., Lambert, J. C., \& Dehnen, W. 2005, MNRAS, 363, 496

Barden, M., Rix, H., Somerville, R. S., et al. 2005, ApJ, 635, 959

Berentzen, I., Athanassoula, E., Heller, C. H. \& Fricke, K.J. 2004, MNRAS, 347, 220

Bertin, E., \& Arnouts, S. 1996, A\&AS, 117, 393

Bolzonella, M., Miralles, J.-M. \& Pelló, R. 2000, A\&A, 363, 476

Bouché, N., Dekel, A., Genzel, R., et al. 2010, ApJ, 718, 1001

Brinchmann, J., Abraham, R., Schade, D., et al. 1998, ApJ, 499, 112

Bruce, V. A., Dunlop, J. S., Cirasuolo, M., et al. 2012, MNRAS, 427, 1666

Bruzual, G., \& Charlot, S. 2003, MNRAS, 344, 1000

Buitrago, F., Trujillo, I., Conselice, C. J., \& Häußler, B. 2013, MNRAS, 428, 1460

Calzetti, D., Armus, L., Bohlin, R. C., et al. 2000, ApJ, 533, 682 
Cameron, E., Carollo, C. M., Oesch, P. A., et al. 2011, ApJ, 743, 146

Conselice, C. J., Bluck, A. F. L., Ravindranath, S., et al. 2011, MNRAS, 417, 2270

Daddi, E., Dickinson, M., Morrison, G., et al. 2007, ApJ, 670, 156

Dickinson, M., Giavalisco, M., \& GOODS Team 2003, in The Mass of Galaxies at Low and High Redshift, ed. R. Bender \& A. Renzini (Berlin:Springer), 324

Förster Schreiber, N. M., Genzel, R., Bouché, N., et al. 2009, ApJ, 706, 1364

Furusawa, H., Kosugi, G., Akiyama, M., et al. 2008, ApJS, 176, 1

Gehrels, N. 1986, ApJ, 303, 336

Giavalisco, M., Steidel, C. C., \& Macchetto, F. D. 1996, ApJ, 470, 189

Giavalisco, M., Ferguson, H. C., Koekemoer, A. M., et al. 2004, ApJ, 600, L93

Grogin, N. A., Kocevski, D. D., Faber, S. M., et al. 2011, ApJS, 197, 35

Hasinger, G., Miyaji, T., \& Schmidt, M. 2005, A\&A, 441, 417

Hayashi, M., Shimasaku, K., Motohara, K., et al. 2007, ApJ, 660, 72

Hozumi, S. 2012, PASJ, 64, 5

Koekemoer, A. M., Faber, S. M., Ferguson, H. C., et al. 2011, ApJS, 197, 36

Law, D. R., Steidel, C. C., Shapley, A. E., et al. 2012, ApJ, 745, 85

Lawrence, A., Warren, S. J., Almaini, O., et al. 2007, MNRAS, 379, 1599

Lilly, S., Schade, D., Ellis, R., et al. 1998, ApJ, 500,75

Martin, D. C., Fanson, J., Schiminovich, D., et al. 2005, ApJ, 619, L1

Melvin, T., Masters, K., Lintott, C., et al. 2014, MNRAS, 438, 2882
Miller, S. H., Bundy, K., Sullivan, M., Ellis, R. S., \& Treu, T. 2011, ApJ, 741, 115

Mortlock, A., Conselice, C. J., Hartley, W. G., et al. 2013, MNRAS, 433, 1185

Noeske, K. G., Weiner, B. J., Faber, S. M., et al. 2007, ApJ, 660, L43

Noguchi, M. 1987, MNRAS, 228, 635

Nonino, M., Dickinson, M., Rosati, P., et al. 2009, ApJS, 183, 244

Ohta, K., Hamabe, M., \& Wakamatsu, K. 1990, ApJ, 357, 71

Ohta, K., Aoki, K., Kawaguchi, T., \& Kiuchi, G. 2007, ApJS, 169, 1

Ostriker, J. P., \& Peebles, P. J. E. 1973, ApJ, 186 , 467

Padilla, N. D., \& Strauss, M. A. 2008, MNRAS, 388, 1321

Patel, S. G., Holden, B. P., Kelson, D. D., et al. 2012, ApJ, 748, L27

Peng, C. Y., Ho, L. C., Impey, C. D. \& Rix, H.-W. 2010, AJ, 139, 2097

Ravindranath, S., Giavalisco, M., Ferguson, H. C., et al. 2006, ApJ, 652, 963

Retzlaff, J., Rosati, P., Dickinson, M., et al. 2010, A\&A, 511, 50

Ryden, B. S. 2004, ApJ, 601, 214

Sargent, M. T., Carollo, C. M., Lilly, S., et al. 2007, ApJS, 172, 434

Sawicki, M. 2012, PASP, 124, 1208

Scarlata, C., Carollo, C. M., Lilly, S., et al. 2007, ApJS, 172, 406

Schade, D., Lilly, S. J., Crampton, D., et al. 1996, ApJ, 451, L1

Sheth, K., Elmegreen, D., Elmegreen, B. G., et al. 2008, ApJ, 675, 1141

Steidel, C. C., Giavalisco, M., Dickinson, M., \& Adelberger, K. L. 1996, AJ, 112, 352 
Talia, M., Cimatti, A., Mignoli, M., et al. 2014, A\&A, 562, A113

Ueda, Y., Akiyama, M., Ohta, K., \& Miyaji, T. 2003, ApJ, 598, 886

van den Bergh, S., Abraham, R. G., Ellis, R. S., et al. 1996, AJ, 112, 359

van der Wel, A., Chang, Y.-Y., Bell, E. F., et al. 2014, ApJ, 792, L6

van Dokkum, P. G., Leja, J., Nelson, E. J., et al. 2013, ApJ, 771, L35

Wang, T., Huang, J.-S., Faber, S. M., et al. 2012, ApJ, 752, 134

Yabe, K., Ohta, K., Iwamuro, F., et al. 2012, PASJ, 64, 60

Yabe, K., Ohta, K., Iwamuro, F., et al. 2014, MNRAS, 437, 3647

Yuma, S., Ohta, K., Yabe, K., Kajisawa, M., \& Ichikawa, T. 2011, ApJ, 736, 92

Yuma, S., Ohta, K., \& Yabe, K. 2012, ApJ, 761, 19

This 2-column preprint was prepared with the AAS LATEX

macros v5.2. 